\title{
Improving the Accuracy of Multi-Valued Datasets in Agriculture Using Logistic Regression and LSTM-RNN Method
}

\begin{abstract}
Abdalla Alameen
Department of computer science, College of Arts and Science, Prince Sattam Bin Abdul Aziz Uninersity, Kharj, Saudi Arabia
\end{abstract}

\begin{abstract}
Changes in environmental factors such as water quality, soil quality, and pollution factors lead to diseases in food producing plants. Identifying plant disease is a very difficult task in agriculture. Plant diseases are also mainly caused by many influences in agriculture which includes hybrid genetics, and the plant lifetime during the infection, environmental changes like climatic changes, soil, temperature, rain, wind, weather etc. The infections may be single or mixed, according to the infections the plants diseases spread. Early detection of plant diseases using recent technologies helps the plants growth. Therefore, Machine Learning techniques are used for early prediction of the diseases. This paper is used to improve the accuracy of detecting plant diseases using the prediction of the soil content in the field land. The techniques Nave Bayes (NB) and Neural Network (NN) were used in the existing system. The proposed system uses Logistic Regression method with Long ShortTerm Memory (LSTM) in Neural Networks (NN) for predicting the soil content and also detects the plant diseases, improves the accuracy level in the plant growth.
\end{abstract}

Keywords - classification, machine learning, neural networks, logistic regression, LSTM.

DOI: 10.18421/TEM111-58

https://doi.org/10.18421/TEM111-58

Corresponding author: Abdalla Alameen,

Department of computer science, College of Arts and Science, Prince Sattam Bin Abdul Aziz Uninersity, Kharj, Saudi Arabia.

Email: a.alameen@psau.edu.sa

Received: 07 November 2021.

Revised: 09 February 2022.

Accepted: 15 February 2022.

Published: 28 February 2022.

(cc) BY-NC-ND (C) 2022 Abdalla Alameen; published by UIKTEN. This work is licensed under the Creative Commons Attribution-NonCommercial-NoDerivs 4.0 License.

The article is published with Open Access at https://www.temjournal.com/

\section{Introduction}

Authors are required basic needs for every living being which are fulfilled by agricultural practice. People's lives depend on agriculture. Agriculture provides food and raw materials to all people in the country. Agricultural people suffer with many issues like decreased productivity, unstable plant growth, different soil moisture, which are considered as the main reason for this decreased productivity. It plays a huge part in agriculture. Due to climatic condition changes, it leads to scarcity of food and everything.

To improve the agriculture production, many methods were implemented. In agriculture, Data mining is one of the recent approaches. Data mining provides essential benefits in making decision on agricultural data. The data mining gains wide knowledge coverage. However, the large data sets are difficult to tackle with multiple number of attributes. Due to above problems, agricultural analysis requires advanced statistical data by expert committee. Recently advancement in Data mining techniques help to build an intelligent data processing in agricultural field [1].

To detect facts in agricultural datasets, a usercentric approach is offered to users. To develop the agricultural quality of plants, managers use confidential information. To predict the growth of the agricultural plant, data mining methods were used. Data mining identifies the relationship between the plant's characteristics and the information in the datasets. Therefore, it gives a productive planting in the agriculture field. From the datasets, it eliminates all the irrelevant data and maximizes the accuracy.

The extraction of relevant data from the plant datasets provides useful information in the growth of plant. In the training datasets, it has unrelated, irrelevant data, and it helps to predict the results. After pre-processing the feature is extracted. The extraction of features, reduces the amount of data. In this research, Artificial Neural Networks (ANN) and Logistic regression were used to improve the accuracy of the plant growth in agriculture. It is a 
statistical analysis method that helps to predict the data values with the help of datasets. Logistic regression is used in machine learning techniques. The algorithm is used for predicting the values and classifies the data sets. One of the supervised classification algorithms is Logistic regression; it is used to predict the target value. The target value is also known as dependent variables. The dependent variables are binary; it might have 1 or 0 . There are many types of logistic regression algorithms such as:

- Binary or Binomial

- Multinomial

- Ordinal

The Artificial neural networks (ANNs) are a system made up of both hardware and software operations that aid neurons in the functioning of the human brain. The capability to maintain data from prior time stamps with help of memory cells are added to the standard feed forward network (FFNN) using recurrent neural network (RNN) [2]. The RNN has good learning capacity and the volumetric prediction of soil water which is done using content by memory cells. The Long short term memory networks (LSTM) is a specially constructed RNN that progressively processes the dynamics of longterm sequences to describe the nonlinear relationship between inputs and outputs. The work contribution is:

1. Soil content prediction using logistic regression in Long Short-Term Memory Network (LSTM).

2. Next, the predicted data is trained and tested using an artificial neural network.

3. The prediction of diseases is done twice, first in logistic regression technique and secondly in artificial neural network.

Further, the document is organized as sections as it is shown. The section 2 consists of brief study of existing agriculture, logistic regression and LSTMRNN. Section 3 discusses the working principal of the proposed model. Section 4 evaluates the result and gives comparison of different algorithms. Section 5 shows the conclusion of the research work with the future scope.

\section{Related Work}

Researchers have developed and used different strategies to increase the effectiveness and accuracy of recent techniques. Each technique has specific weaknesses for predicting plant diseases, such as offering a particular classification model with prognostic power, low production efficiency with increased data volume and best consistency. Similarly, due to result production with too many false positives, most techniques are not suitable for real environment. A false positive indicates a false identification of algorithm used for classification.

Data mining offers important benefits to agriculture-based approach and decision-making. The technology field of data mining has received good coverage. However, it is difficult to influence terribly gigantic data sets with many attributes. Here, sophisticated statistical analysis is used by agricultural experts in the field. Processing techniques expand the possibilities and help as an open source of knowledge processing for agriculture [3]. Logistic regression is the most popular algorithms in Machine learning techniques. It comes under the technique of supervised learning. It is used for predicting the output of dependent variable.

The dichotomous of target individual has two classes of capacity. In flowing sentences, the established variable with binary values, with comprehension coded as one (means achievement/sure) or 0 (means failure/no) is presented. Mathematically, a presentation predicts regression as $\mathrm{P}(\mathrm{Y}=1)$ as a realization of $\mathrm{X}$. This cc algorithm is considered as best and used in class based problems such as unsolicited mail detection, polygenic disease prediction, cancer detection and many more.

Logistic regression is one of the machine learning classification algorithms for categorical values such as Yes or No, True or False, 0 or 1. It is a verified classification.

There are many processor in ANN. It computes parallel in all layers. The information in the first layer is raw input data. The input of next layer is output of previous layer. An example of processing data, biological nerve technique is activated, similar as brain information technique, which is Artificial Neural Networks (ANN).

The enormous neurons are interconnected for processing in unity to solve a particular task in neuron [4]. This simple networking sense is formed with excessive interrelated data processing directly with related task which is executing. The processing unit are interconnected and connections are underlying in it. The results are efficiently provided by neural network technique in spite of low significant variables. The Feed Forward and Recurrent Neural Networks are two types of neural techniques used here [5].

The output of the system is given in the last layer. Each process node has its own domain of knowledge, as well as what it saw and initially some rules are programmed or developed for itself. The layer is interconnected extremely, suggesting that layer $n$ nodes in each neural network will connect to multiple nodes in layer $\mathrm{n} 1$ at its entrances and $\mathrm{n}+1$ layers, which provides an input file for these nodes. The output layer has one or more nodes, which produces the solution to read. 
Worldwide, irrigation is planned and based on visual inspection of crops by farmers, and as a result, approximately $50 \%$ of water is wasted by using traditional irrigation techniques [6]. Control based irrigation techniques are reminiscent of sprinkler, drip and pre irrigations techniques reduce wastage of water by $3075 \%$ [7]. So, there is demand for feedback about the irrigation system with built-in accuracy to use water quickly without harming crop development. Accurate irrigation requires moisturizing soil, information on climate, depth of drip and type of crop to calculate the correct quantity of water and amount of irrigation required to improve crop yield, reducing labor cost of farmer.

The platform provided by Internet of Things (IoT) for practicing good farming, wireless connectivity for many soil sensors is done. They are all completely different hardware (e.g., water pumps, sprinklers, management, etc.) and applications are analyzed to increase the ability of farmer to solve advanced agricultural problems like land cleaning, water requirement estimation, yield forecasting, etc. during the growth and harvest cycle [8].

Today, data driven approach with machine learning (ML) techniques for irrigation [9] have become more preferred over physical/mechanical models [10] for predicting soil water content due to the knowledge that less data is needed for normalization in comparison with calibration of the mechanistic model. In addition, machine learning models enable time-based and simple data to be extracted in less time and display sensitive prophetic performance compared to physical models.

Irrigation systems based on a machine learning model has controller of closed loop which study from calibration, where the system uses feedback from the pre-processed information and the data from the measured period. In [11], [12] the authors proposed a fuzzy call system to volumetrically predict soil water content to estimate optimal water demand, while in [13] a neural network model was implemented for calculating threshold price based on water usage of levels. Existing machine learning approaches show sensitive result compared to other models, but does not support various environmental situations.

These techniques are limited to some regions in which it uses data label from regions. Here the intelligence processed in limit to $\mathrm{NN}$ for losing data from previously processed data. The Recurrent Neural Network with Feed Forward Neural Network introduces flexibility to keep data safe from previous steps with the help of memory cells [11]. Soil meter predicts its moisture and this data helps RNN to process accurately. The short-term memory has RNN in it for representing the relationship between input and outputs non linearly through a sequential dynamically for long time [15]. In [16], deep learning technique is proposed by authors to predict moisture content in the context of passive active with of NASA's soil moisture project.

In [17], the authors discuss hydrological LSTM model for temporal dynamics prediction, which is supported by depth of the irrigation: diversion of water, precipitation, temperature, and evaporation. In [18], LSTM relevant technique is proposed to show the performance higher than the standard model of neural network.

Logistic regression is commonly used once the variables are not ordinarily distributed, and a few of them is also categorical. The spatial prediction relies on dependent and independent variables that are accessible across the region in an exceedingly spatially certain method or linear direction. The basic hypothesis is that a selected dependent variable is classed as a binary variable by logistic regression (i.e., presence, absence, etc.). Therefore, the logistic regression predicts the likelihood of presence and absence variables and offers the predictor variable as observed value [19]. Depending at the hardware, the tool desires effective processors which can do parallel processing paintings effectively. This device is more able to tolerate the faults. In the idle country one or greater neurons now have longer effect on the resulting, and the output of processes had been acquired from produced neutrons [14].

\section{Proposed Logistic Regression and LSTM- RNN Method}

The existing prediction system uses classification and single prediction method to improve the accuracy of detecting the plant growth. The diseases in the plant stop the growth of the plant. It only implies the growth of the plant. The plant growth depends on various factors like soil, climatic conditions, and water. In the proposed system, logistic regression with LSTM in neural networks, gives classification and prediction methods. Therefore, the growth of the plant and soil content is also improved in the proposed system.

\subsection{Types of Plant Diseases}

In this proposed system, Soybean datasets were used for classification method. There are many types of diseases which occurred in the soybean plant like Bacterial diseases, Anthracnose, Brow stem root, Downy Mildew, Septoria brown spot, Soybean rust and many other fungal and viral diseases, too. These diseases are identified from the plants and then they are classified. According to the diseases the accuracy varies. The first step is detecting the diseases, followed by training and testing, and then the essential solution for improving production and growth is given to the farmer. 


\subsection{Overview of Logistic Regression and LSTM- RNN Method}

In this proposed methodology, the hybrid of neural networks and logistic regression with LSTM algorithms were used to improve the accuracy of plant growth, and they are used for predicting the soil content in the agriculture field. Figure 1 shows the overall process.

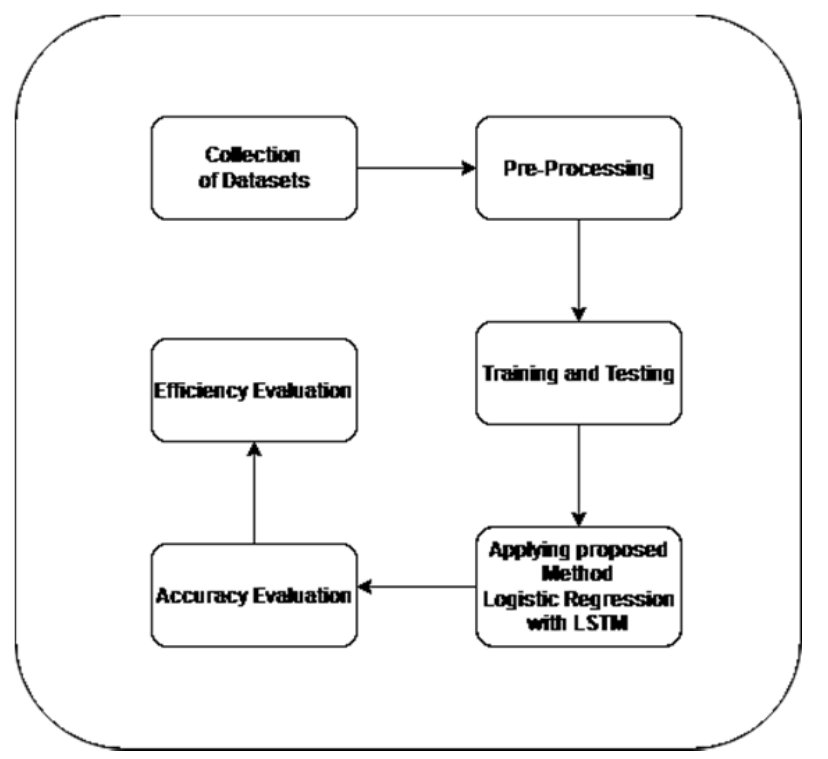

Figure 1. Proposed architecture for plant diseases prediction

The overall architecture is explained below which is shown in Figure 1. The soil dataset is collected and unwanted noises are removed using pre-processing step. Prediction of diseases is done using logistic regression with LSTM in neural networks. The detailed discussion of the prediction system is given in following section.

Collection of Datasets: There are many agricultures online repositories which are available today. Datasets were collected from the UCI repository. In those datasets, there is information about soybean, eucalyptus, squash and white-clover plants. These datasets were used in the entire process.

Pre-processing: Pre-processing is the first step; it is used for removing the irrelevant data. It removes the noisy or incomplete data from the collected datasets. The step-in pre-processing contains:

- Fills the Missing data:

Decline the tuple.

Manually, fills all the missing values.

It uses Central Tendency, Median.

Fills with probable values.

- Removing Noisy Data: Basically, random errors may call as Noisy data. It helps to identify the outliers in the datasets and removes the inconsistencies of the data. In this paper, some of the pre-processing techniques were used, and they are:

i. $\quad 10$-Fold cross validation method is used for testing and training.

ii. For feature selection, BBFS and CFS were used to optimize the data.

- Testing and Training: Separating the data into testing and training sets from the collected datasets is one of the important parts of evaluation. While separating the data sets, most of the data is used for training data sets, and remaining part is used for testing sets.

\subsection{LSTM in Neural Networks}

$\mathrm{RNN}$ is a feed forward neural network; it has its own internal memory. To process the order of entries, RNN uses its internal memory. The LongShort Term Memory Network (LSTM) is particularly planned for the Recurrent Neural Network (RNN). It helps to remember the data in memory, Figure 2.

The proposed model has components which helps the user (farmer) to take decision about the irrigation land. The components were explained below

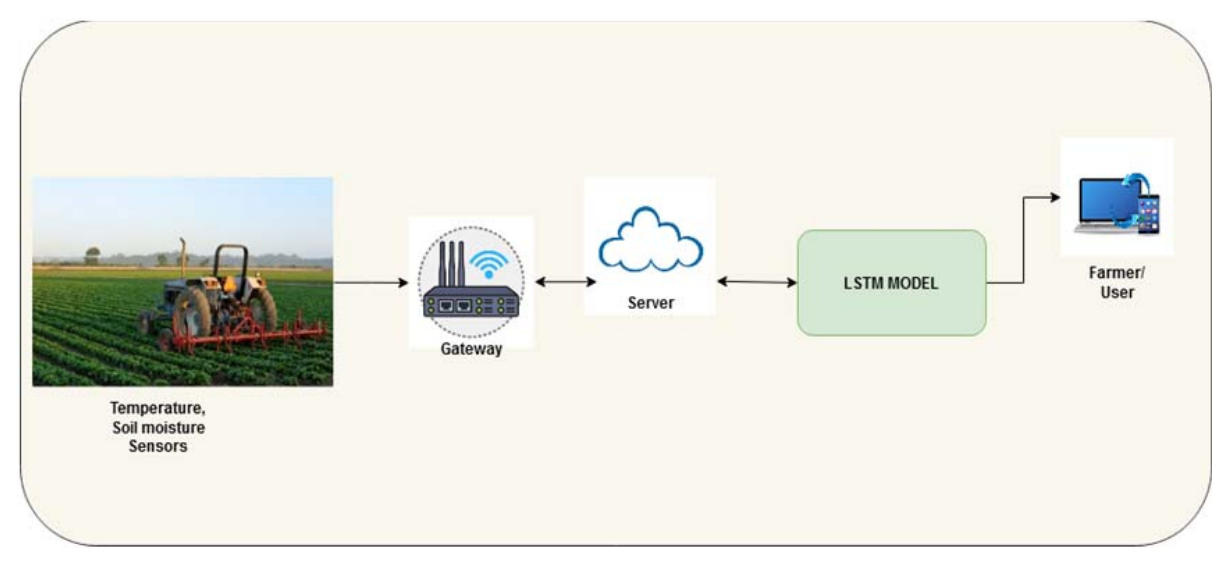

Figure 2. Irrigation using LSTM network model 
- First, it has set of nodes i.e., soil sensors, raingauge sensors. With the help of wireless sensor nodes, it measures the air, temperature, soil moisture, soil temperature.

- Second, the nodes were connected to the gateway. Therefore, it receives data from the field with the help of sensors and then forwards to the server for storage.

- Third, LSTM RNN model, predict the soil moisture and soil information. The output from the model is given to the user for irrigation. With the help of smart phone, the end user receives all the information about the irrigation land.

\subsection{LSTM-RNN Model}

Here, hydrological aspect of the LSTM and RNN model is predicted to volumetrically predict soil moisture content one day in advance. The LSTM network covers hydrological aspect to the memory of the LSTM-RNN like soil water content, soil temperature, precipitation level, air temperature and humidity for the prediction of the soil water content for a day ahead.

The input temporary data of LSTM-RNN as $=\left[I_{1}\right.$, $\left.I_{2} \ldots, I_{\mathrm{t} . .} I_{\mathrm{k}}\right]$ consisting $\mathrm{k}$ days consecutively for linear parameters independently, and side by side input is processed in the units of the LSTM network memory to successfully predict the water content in soil per day in output as $S M(t+1)$. At the moment $(1 \leq t \leq$ $K)$, it contains current input vector data received at detector nodes, i.e., $I t=[\mathrm{a \mp}(t), \mathrm{aH}(t), S M(t), S \mp$ $(t), R L(t)]$ is processed using LSTM network layer with its memory unit. The values aҒ $(t), S \mp(t), S M$ $(t), S \mp(t)$ and $R L(t)$ are for humidity in air, moisture of soil, temperature and rainfall in day time, respectively. For an economic training of the expected LSTM RNN model, all discovered input files and output data are normalized, which can be done by subtracting the average from the daily data and quality deviation which is divided using results, each time the mean and variance are computed from the standardization training.

\section{Algorithm 1: LSTM RRN algorithm}

Input: $\mathrm{I}=\{1, \ldots \mathrm{K}\}$ days

Output: $\operatorname{sm}$ (soil moisture) \#predicting the soil moisture content\#

1. Memory size is initialized and maximizes the episode $\mathrm{s}$

2. Begin the coefficient weights wt

3. Initialize $\hbar 0, \mathbb{C} 0=0$ of length $\mathrm{K}$

4. For $E p=1, \ldots \ldots$ Epmax

5. Sample the mini batch with size $2_{\mathrm{r}}$

6 . For each $t=1,2 \ldots \ldots \mathrm{K}$

7. Fetch sample for mini batch
$I t=[\mathrm{a} F(t), \mathrm{aH}(t), \operatorname{sm}(t), \mathrm{s \mp}(t), \mathrm{r} L(t)]$ and $\mathrm{Y}_{o b s}$

8. Calculate the equation

9. Cell memory state is update

10. Calculate equation

11. End for

12. Output: $\hbar=\{\hbar 1, \ldots \ldots \hbar \mathrm{K}\}$

13. Predicted output is calculated

14. Evaluate loss function

15. LSTM weight parameter is updated

16. End for

\#Prediction\#

17. Examine and collect the environment and information of the land

$I t=[\mathrm{a} F(t), \mathrm{aH}(t), \operatorname{sm}(t), \mathrm{s} \mp(t), \mathrm{r} L(t)]$

18. Input is given into the trained LSTM model

19. Soil moisture content $\operatorname{sm}(t+1)$ is predicted

20. The input is stored into the tuple in replay memory set.

The Algorithm 1 shows the proposed method framework. The input values $I t=[\mathrm{a \mp}(t), \mathrm{aH}(t)$, $\operatorname{sm}(t), \mathrm{s} \mp(t), \mathrm{r} L(t)]$ are given into the network which is trained for volumetric prediction using soil moisture $\operatorname{sm}(t+1)$ content as of later by one day. The volumetric soil water content prediction using the crop data (root growth) and soil data for estimating the irrigation timing and the required amount of water for crop growth is done ultimately.

\subsection{Logistic Regression}

The individual of goal or variable is dichotomous, which implies that there might be only 2 capability classes. In smooth phrases, the established variable is binary in nature having understood coded as either one (stands for fulfilment/sure) or 0 (stands for failure/no). Mathematically, a presenting regression model predicts $\mathrm{P}(\mathrm{Y}=1)$ as a carry out of $\mathrm{X}$. It is one in all the best cc algorithms that can be used for numerous class issues adore unsolicited mail detection, polygenic disease prediction, cancer detection and many others.

Logistic regression is one of the classification algorithms in machine learning for categorical values like Yes or No, True or False, 0 or 1. It is a supervised classification algorithm.

\section{Types of Logistic Regression}

Typically, logistic regression indicates that binary logistic regression is having binary target variables. However, there is two additional classes of target variables that should be foretold through it. Supported the ones range of classes, provision regression can be divided into following varieties. 


\section{Binary or Binomial}

In any kind of such a classification, a variable can have solely two practicable kinds both one and zero. As an example, those variables may additionally represent success or failure, affirmative or no, win or loss and so forth.

\section{Multinomial}

In such a kind of category, variable may have 3 or extra attainable unordered sorts or the types having no quantitative importance. For instance, those variables might constitute "type A" or "kind B" or "kind C".

\section{Ordinal}

In the sort of form of class, based variable will have 3 or greater possible ordered types or the kinds having a quantitative importance. As an example, those variables can also constitute "terrible" or "accurate", "superb", "extremely good" and every class will have the rankings like $0,1,2,3$.

\subsection{Neural Networks}

Artificial Neural Networks (ANN) is a computational model and is made by human brain and it is composed of neurons. Neural network's basic unit is neuron or node. An ANN has large number of processors; it is operated in the parallel mode and arranged in layers. The ANN has three layers; they are input, hidden and output layers. The first layer obtains the unprocessed input information. Every layer obtains the output from the layer preceding, and it is hidden layer. The system receives output from last layer. Each process node has its own area of knowledge, as well as what it saw and all the rules it initially programmed or developed for itself. The interconnected layers suggest that each node $n$ connected to multiple nodes in layer $\mathrm{n} 1$ at its entrances and in layer $\mathrm{n}+1$, which provides an input file for these nodes. There may also be one or more nodes in the output layer, from which the solution it produces can be read. Figure 3 shows the layer of neural networks.

Neural networks are usually represented in terms of depth, with the percentage of layers they need between input and output, or the questionable hidden layers of the model. Perhaps this is why the term neural network is practically used as a synonym for deep learning. They are even described by the number of hidden nodes in the model or by the number of inputs and outputs of each node.

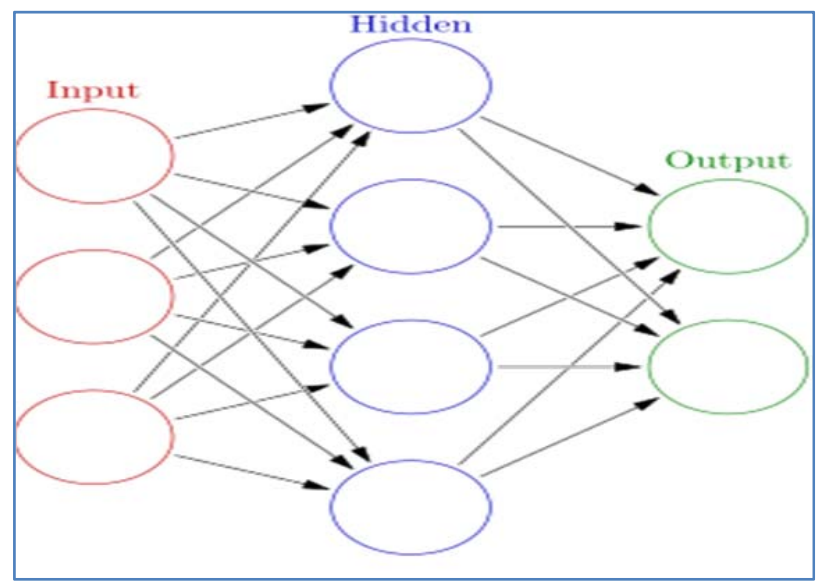

Figure 3. Layers of neural networks

\section{Result Analysis}

The proposed method uses different methodologies to improve the accuracy of the plant growth. The dataset was used in this method were taken from the UCI repository. Table 1 shows Logistic Regression and LSTM-RNN in Soybean dataset. Comparing with the existing system, the proposed method uses the metrics such as accuracy, precision, recall and fmeasure. It is proposed to classify a sample as positive when it is correctly classified TP (true positive) and FN (false negative) if it is classified as negative. TN (True Negative) means that a negative sample is classified as negative while the opposite is FP (False Positive).

Precision: The precision is calculated using the correctly classified positive crops to the total positives in the dataset. The below equation is used:

$$
\text { Precision }=\frac{\mathrm{TP}}{\mathrm{FP}+\mathrm{TP}}
$$

Figure 4 shows the proposed Logistic regression and LSTM-RNN method the precision is calculated. The number of datasets is compared, and the graph shows the precision for the datasets. When the datasets are greater the precision value is greater. The proposed Logistic regression and LSTM-RNN has high precision values of $95.53 \%$ compared with MLP and Logistic regression methods. 


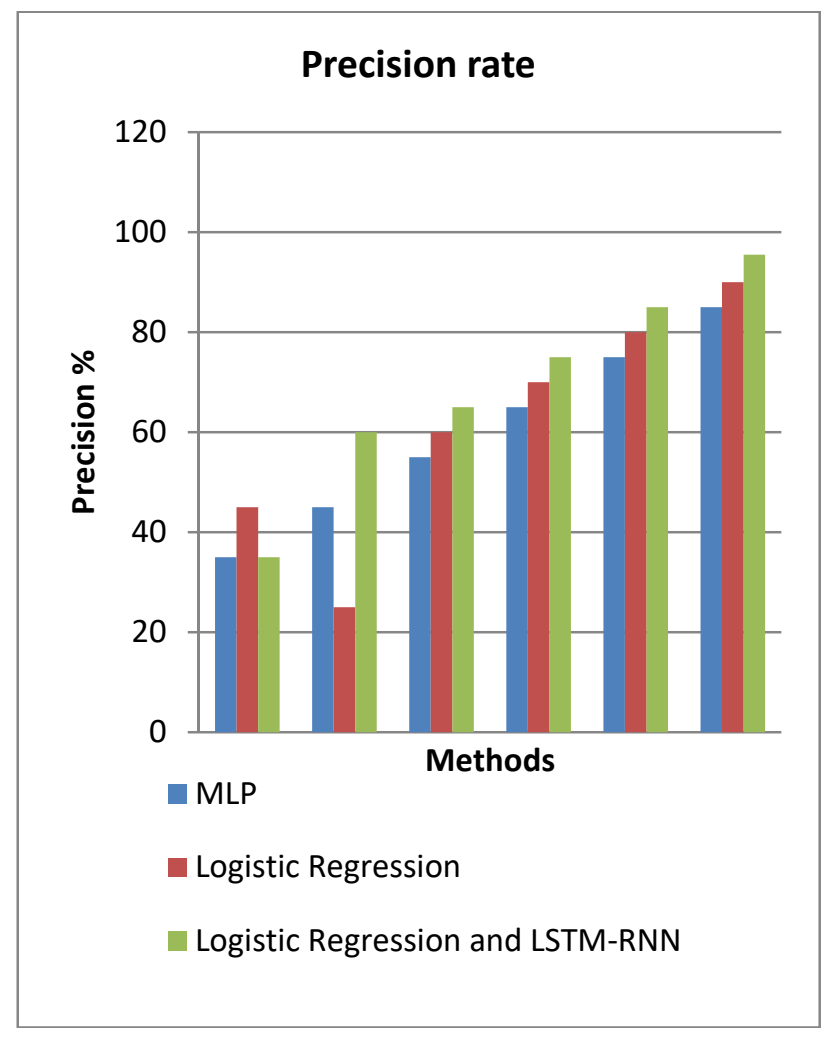

Figure 4. Precision rate results

Recall: The correctly classified crops to total count of positive samples are calculated by the equation:

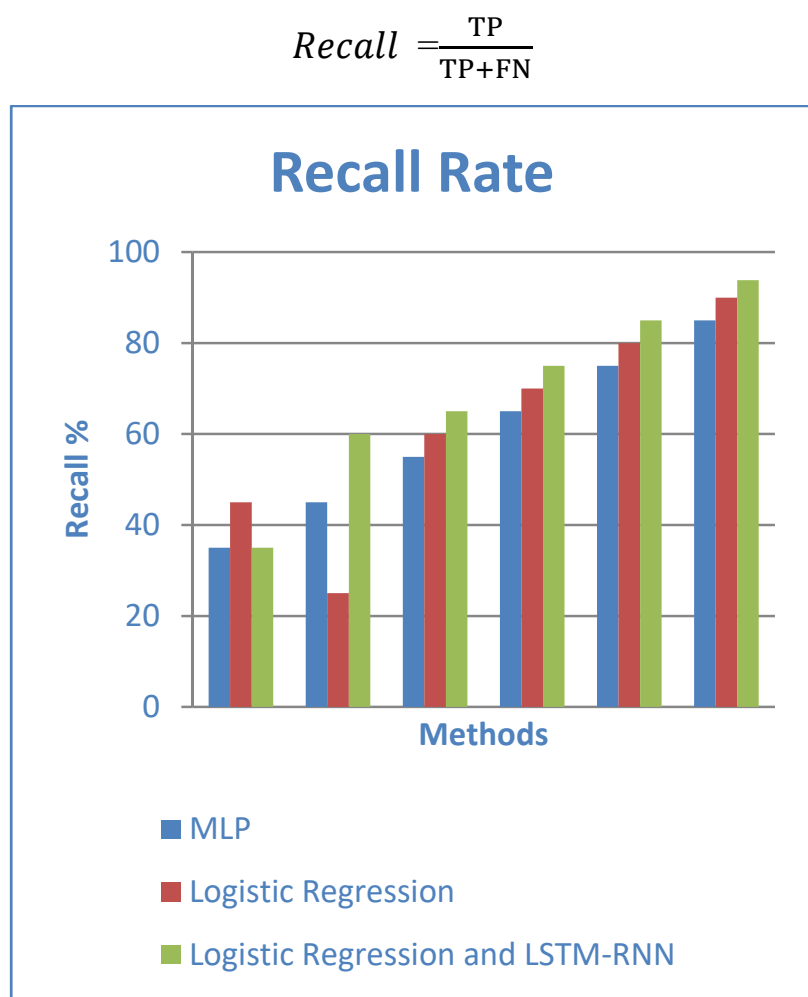

Figure 5. Recall rate results

Figure 5 shows the proposed Logistic regression and LSTM-RNN methods calculated the Recall; the graph shows the number of datasets in specified datasets. When the number of datasets is greater the recall is greater. The proposed Logistic regression and LSTM-RNN has high Recall values of $93.83 \%$.

F-measure: $F 1$-score is also known as $\mathrm{F}$-measure. It is the harmonic mean of precision and recall is calculated by the equation:

$$
F-\text { measure }=\frac{2 * \text { Recall } * \text { Precision }}{(\text { Recall }+ \text { Precision })}
$$

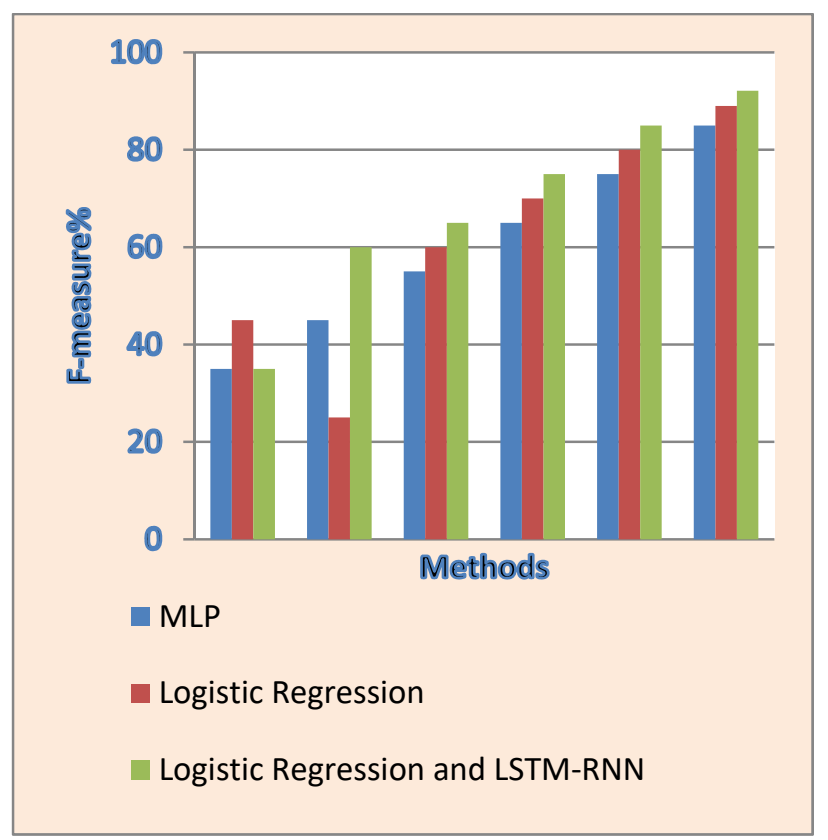

Figure 6. F-measure results

Figure 6 shows the proposed Logistic regression and LSTM-RNN methods the graph gives the details about the datasets. The proposed Logistic regression and LSTM-RNN has high F-measure values of 92.13 $\%$.

Accuracy: It is calculated by the ratio between correctly classified crop samples to total crop samples and the following equation is used for calculation:

$$
\text { Accuracy }=\frac{\mathrm{TP}+\mathrm{TN}}{\mathrm{TP}+\mathrm{TN}+\mathrm{FP}+\mathrm{FN}}
$$

Table 1. Logistic regression and LSTM-RNN in soyabean dataset

\begin{tabular}{|l|c|c|}
\hline \multirow{2}{*}{ Forecasting Practice } & \multicolumn{2}{|c|}{ Attain Accuracy (\%) } \\
\cline { 2 - 3 } & $\begin{array}{c}\text { Full } \\
\text { Features }\end{array}$ & $\begin{array}{c}\text { Optimal } \\
\text { Features }\end{array}$ \\
\hline $\begin{array}{l}\text { Multi-Level } \\
\text { Perceptron(MLP) }\end{array}$ & 93.66 & 93.88 \\
\hline Logistic Regression & 93.15 & 93.01 \\
\hline $\begin{array}{l}\text { Proposed Logistic } \\
\text { Regression and } \\
\text { LSTM-RNN }\end{array}$ & 98.26 & 97.37 \\
\hline
\end{tabular}

In Table 1 many algorithms were compared, thus the proposed logistic regression and ISTM-RNN gives more accuracy level in the full features and optimal features. In the existing system the accuracy range is minimum. 
Thus, the Table 2 shows the different algorithms and datasets were used, the proposed Logistic regression and LSTM-RNN gives better accuracy than the existing systems.

Table 2. Dr.Geraldin B.Delacruz soyabean dataset

\begin{tabular}{|l|c|}
\hline \multicolumn{1}{|c|}{ Forecasting Practice } & Accuracy (\%) \\
\hline Multi-Level Perceptron(MLP) & 93.66 \\
\hline Logistic Regression & 93.15 \\
\hline Dr. Geraldin B.Dela Cruz(11) & 95.11 \\
\hline LSTM-RNN & 98.26 \\
\hline
\end{tabular}

The results were shown in the above tables, thus the proposed method achieved high predictive accuracy of soybean, while compared with the Naive bayes algorithms. Figure 7 shows the results of the soybean dataset with several algorithms. The proposed system achieves more accuracy than the existing system.

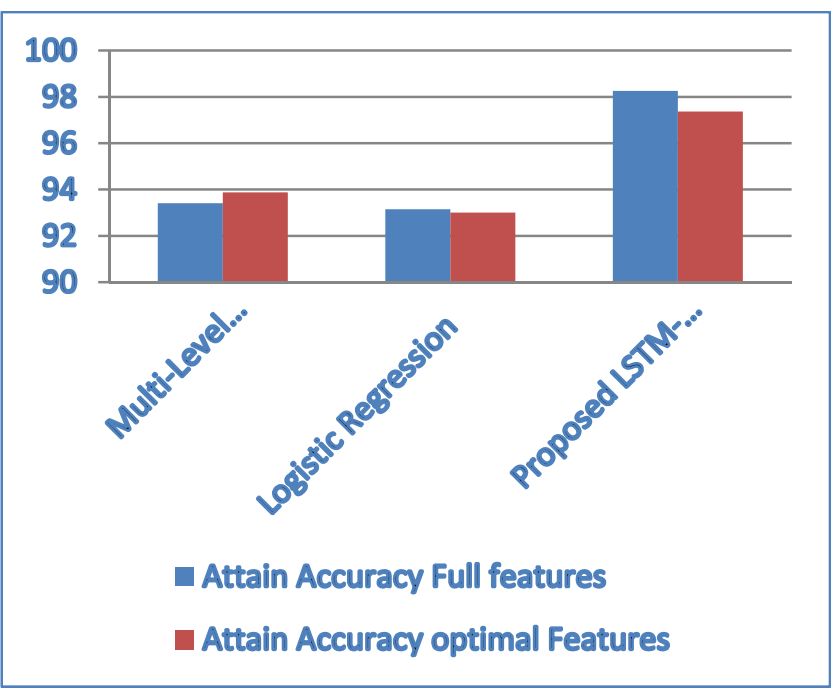

Figure 7. Proposed logistic regression and LSTM-RNN among soybean dataset

\section{Conclusion}

In the proposed paper, classification of disease and soil moisture content prediction was conducted for the improvement in the agriculture field. For high yield, the diseases are identified first, and the soil moisture content should be predicted. Therefore, the proposed algorithms were effectively used, and it gives high accuracy level and predicts the soil moisture content i.e., the water level in the soil. With the help of sensors, the soil moisture is predicted, thus the farmer can sprinkle the water whenever they needed. So, the proposed system identifies the diseases of the plant and identifies the soil moisture level in the land. After these predictions, the farmers can give more yields in their land. It reduces the time and workers. Thus, the proposed system Logistic regression and LSTM-RNN computes the diseases present in the leaf, and also it shows how much pesticides should be used in the field. Also, it rectifies the problems with low cost and time and produces more accuracy.

In future, in the agriculture field, many machine learning techniques will be implemented. So that, there is an increase in the plant growth. Also, many prediction methods should be used to improve the soil moisture level.

\section{References}

[1]. Agrawal, A., Patel, M., \& Sharma, A. K. (2021, January). Novel Supervised Machine Learning Classification Technique for Improve Accuracy of Multi-Valued Datasets in Agriculture. In 2021 6th International Conference on Inventive Computation Technologies (ICICT) (pp. 1067-1070). IEEE.

[2]. Kashyap, P. K., Kumar, S., Jaiswal, A., Prasad, M., \& Gandomi, A. H. (2021). Towards precision agriculture: IoT-enabled intelligent irrigation systems using deep learning neural network. IEEE Sensors Journal, 21(16), 17479-17491.

[3]. Raman, M., Sharma, V. K., Hiranwal, S., \& Bairwa, A. K. (2021). Efficient method for prediction accuracy of heart diseases using machine learning. In Proceedings of International Conference on Communication and Computational Technologies (pp. 113-121). Springer, Singapore.

[4]. Joshi, S., \& Patel, M. Natural language processing for classifying text using Naïve Bayes model. Paideuma J, 13(10), 72-77.

[5]. Patel, M., Badi, N., \& Sinhal, A. (2019). The role of fuzzy logic in improving accuracy of phishing detection system. International Journal of Innovative Technology and Exploring Engineering, 8(8), 31623164.

[6]. Mulenga, R., Kalezhi, J., Musonda, S. K., \& Silavwe, S. (2018, June). Applying Internet of Things in monitoring and control of an irrigation system for sustainable agriculture for small-scale farmers in rural communities. In 2018 IEEE PES/IAS PowerAfrica (pp. 1-9). IEEE.

[7]. Parra-Boronat, L., Rocher-Morant, J., García-García, L., Lloret, J., Tomás Gironés, J., Romero Martínez, J. O., ... \& Roig-Merino, B. (2018). Design of a WSN for smart irrigation in citrus plots with fault-tolerance and energy-saving algorithms. Network Protocols and Algorithms, 10(2), 95-115.

[8]. Navarro-Hellín, H., Martinez-del-Rincon, J., Domingo-Miguel, R., Soto-Valles, F., \& TorresSánchez, R. (2016). A decision support system for managing irrigation in agriculture. Computers and Electronics in Agriculture, 124, 121-131.

[9]. Chrouta, J., Chakchouk, W., Zaafouri, A., \& Jemli, M. (2018). Modeling and control of an irrigation station process using heterogeneous cuckoo search algorithm and fuzzy logic controller. IEEE Transactions on Industry Applications, 55(1), 976990. 
[10]. Giusti, E., \& Marsili-Libelli, S. (2015). A fuzzy decision support system for irrigation and water conservation in agriculture. Environmental Modelling \& Software, 63, 73-86.

[11]. King, B. A., \& Shellie, K. C. (2016). Evaluation of neural network modeling to predict non-waterstressed leaf temperature in wine grape for calculation of crop water stress index. Agricultural water management, 167, 38-52.

[12]. Park, Y., Shamma, J. S., \& Harmon, T. C. (2009). A Receding Horizon Control algorithm for adaptive management of soil moisture and chemical levels during irrigation. Environmental Modelling \& Software, 24(9), 1112-1121.

[13]. McCarthy, A. C., Hancock, N. H., \& Raine, S. R. (2014). Simulation of irrigation control strategies for cotton using model predictive control within the VARIwise simulation framework. Computers and electronics in agriculture, 101, 135-147.

[14]. Khan, T., Sherazi, H. H. R., Ali, M., Letchmunan, S., \& Butt, U. M. (2021). Deep Learning-Based Growth Prediction System: A Use Case of China Agriculture. Agronomy, 11(8), 1551.
[15]. Pietrzykowski, M., \& Sałabun, W. (2014). Applications of Hidden Markov Model: state-of-theart. Int. J. Comput. Technol. Appl, 5, 1384-1391.

[16]. Brezak, D., Bacek, T., Majetic, D., Kasac, J., \& Novakovic, B. (2012, March). A comparison of feedforward and recurrent neural networks in time series forecasting. In 2012 IEEE Conference on Computational Intelligence for Financial Engineering \& Economics (CIFEr) (pp. 1-6). IEEE.

[17]. Zhang, J., Zhu, Y., Zhang, X., Ye, M., \& Yang, J. (2018). Developing a Long Short-Term Memory (LSTM) based model for predicting water table depth in agricultural areas. Journal of hydrology, 561, 918929.

[18]. Zhang, D., Lindholm, G., \& Ratnaweera, H. (2018). Use long short-term memory to enhance Internet of Things for combined sewer overflow monitoring. Journal of hydrology, 556, 409-418.

[19]. Fang, K., Shen, C., Kifer, D., \& Yang, X. (2017). Prolongation of SMAP to spatiotemporally seamless coverage of continental US using a deep learning neural network. Geophysical Research Letters, 44(21), 11-030. 